\title{
Self-organized growth of cluster arrays
}

\author{
K. Bromann ${ }^{1}$, M. Giovannini ${ }^{1}$, H. Brune ${ }^{1, a}$, and K. Kern ${ }^{1,2}$ \\ ${ }^{1}$ Institut de Physique Expérimentale, EPFL, CH-1015 Lausanne, Switzerland \\ ${ }^{2}$ Max-Planck-Institut für Festkörperforschung, Heisenbergstrasse 1, D-70569 Stuttgart, Germany
}

Received: 2 September 1998 / Received in final form: 27 January 1999

\begin{abstract}
We present a novel method for the fabrication of well-ordered, two-dimensional nanocluster arrays. The method is based on the confined nucleation of adatoms within the superstructure cells of periodic surface dislocation networks, which form in many heteroepitaxial systems. We show how quantitative understanding of adatom diffusion and heterogeneous nucleation on such surfaces can be obtained through kinetic Monte-Carlo simulations and discuss the potential of this approach.
\end{abstract}

PACS. 61.46. $+\mathrm{w}$ Clusters, nanoparticles, and nanocrystalline materials - 81.15.Hi Molecular, atomic, ion, and chemical beam epitaxy - 68.65.+g Low-dimensional structures (superlattices, quantum well structures, multilayers): structure, and nonelectronic properties

During the last years, much effort has been devoted to the fabrication of nanostructures on semiconductor and metal surfaces. Research first focused on the variety of structures that could be produced, either by serial techniques such as atom manipulation with the tip of a scanning tunneling microscope (STM) [1], or by parallel processes such as self-organized growth [2-5]. Recently, the interest turned towards the problem of increasing homogeneity and spatial regularity of the nanostructure populations. The controlled deposition of size-selected clusters from the gas phase [6], e.g., produces nanostructures of nearly uniform dimensions. But, like the approach of self-organized growth, this method suffers from the statistics inherent in deposition and leads to largely uncorrelated spatial distributions. Improved lateral order was obtained by exploiting the spatial correlation of island nucleation in a sequence of island and spacer layers in semiconductor superlattices [7]. In a different approach, the preferred nucleation of $\mathrm{Ni}$ at the elbows of the $\mathrm{Au}(111)$ herringbone reconstruction resulted in ordered lines of islands [8], which later could be explained with a site-specific exchange process for this specific system [9].

In the present contribution we present a method to fabricate ordered arrays of equally spaced nanostructures that is of potential applicability in a large variety of systems. On substrates with a periodic arrangement of dislocations, regular superlattices of almost monodispersed islands can be created by self-organized growth. Due to mutual long range repulsions the dislocations arrange into highly ordered periodic patterns that can be transferred into nanostructure superlattices through heterogeneous nucleation. The mechanism imposing the inhomogeneous substrate structure on the nucleation and growth process

\footnotetext{
a Corresponding author. e-mail: harald.brune@epfl.ch
}

is the strong repulsion of dislocations towards diffusing adatoms. Here, we illustrate this method for Ag nucleation on the second monolayer (ML) of $\mathrm{Ag}$ on a $\mathrm{Pt}(111)$ substrate (see Fig. 1b). The method, however, is of general importance as the required periodic substrates are provided by numerous epitaxial systems that show strain relief through dislocation formation. So far, besides the above mentioned Ag cluster arrays, we applied the method successfully to arrays of Co clusters on $2 \mathrm{ML}$ of $\mathrm{Cu}$ on $\mathrm{Pt}(111)$ and Fe clusters on the same substrate [10].

The experiments were performed with a variable temperature STM operating in UHV. The accessible temperature range for in situ measurements is $25-800 \mathrm{~K}$ [11]. $\mathrm{Ag}$ was evaporated from a Knudsen cell with a flux of $1.1 \times 10^{-3} \mathrm{ML} / \mathrm{s}$. The STM images were taken at the deposition temperature, with the exception of Fig. 1a, which was taken at $T=300 \mathrm{~K}$.

Figure 1a shows the growth template, the equilibrium structure of the second monolayer of $\mathrm{Ag}$ on $\mathrm{Pt}(111)$. The lattice constant of $\mathrm{Ag}$ is $4.3 \%$ larger than the one of $\mathrm{Pt}$. In the second Ag-monolayer, the compressive strain resulting from this lattice mismatch is relieved in a trigonal network of dislocations with a periodicity of 25 atoms [12]. The dislocations form transitions from fcc to hcp stacking. In contrast to comparable reconstructions, where dislocations run parallel, here the dislocations cross each other allowing for isotropic strain relief on a small length scale. Larger areas of the energetically favored fcc stacking with respect to hcp stacking areas are achieved by displacing one class of domain walls by an offset $\delta$ (inset Fig. 1a) relative to the crossing point of the two others. This results in a pattern with trigonal symmetry. Each unit cell shows a large quasi hexagon with fcc stacking and two triangles of different size with hcp stacking; for details of this strain relief pattern the reader is referred to [12]. 
a)

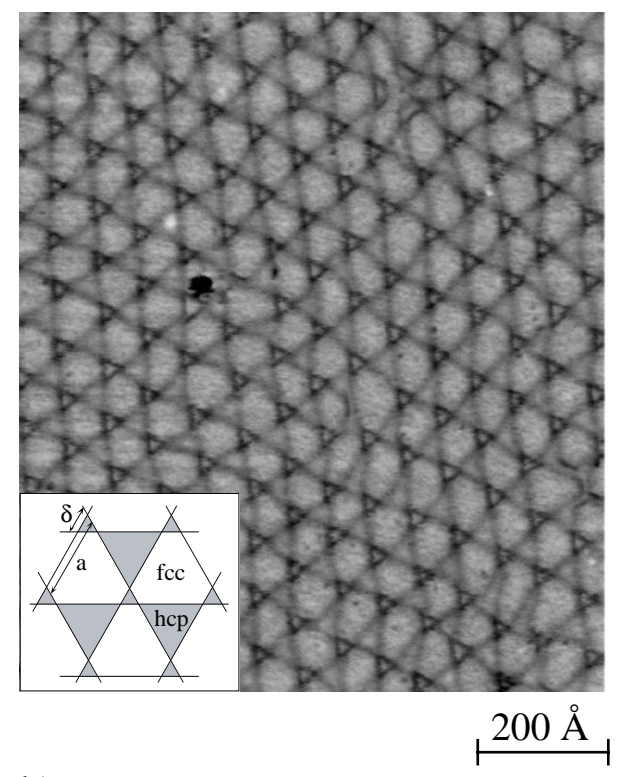

b)

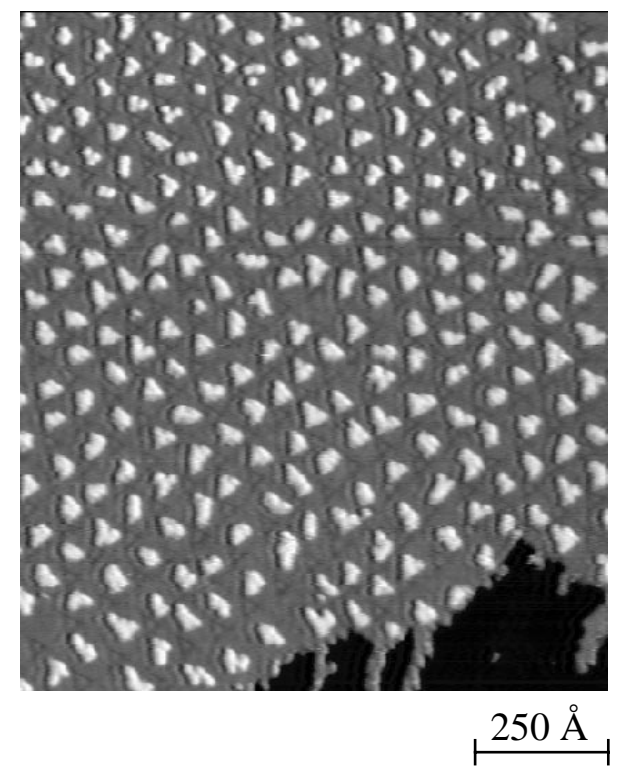

Fig. 1. (a) STM image of the $(25 \times 25)$ dislocation network formed by the second $\mathrm{Ag}$ monolayer on $\mathrm{Pt}(111)$ upon deposition at $400 \mathrm{~K}$ and subsequent annealing to $800 \mathrm{~K}$. The inset shows a model of this trigonal strain relief pattern. (b) A superlattice of islands is formed upon $\mathrm{Ag}$ deposition onto this network at $110 \mathrm{~K}$ (coverage $\theta=0.10 \mathrm{ML}$ ).

Ag nucleation onto this network at low temperature leads to a high density of islands (Fig. 2a). Only a few of these islands are located near the dislocations. This implies that dislocations constitute repulsive barriers towards diffusing adatoms. With increasing temperature, the island density approaches a stationary value between $100 \mathrm{~K}$ to $130 \mathrm{~K}$, where exactly one island forms per network unit cell (see Figs. 1b and 2b). All of the islands nucleate within the distorted hexagons. This implies preferential binding to
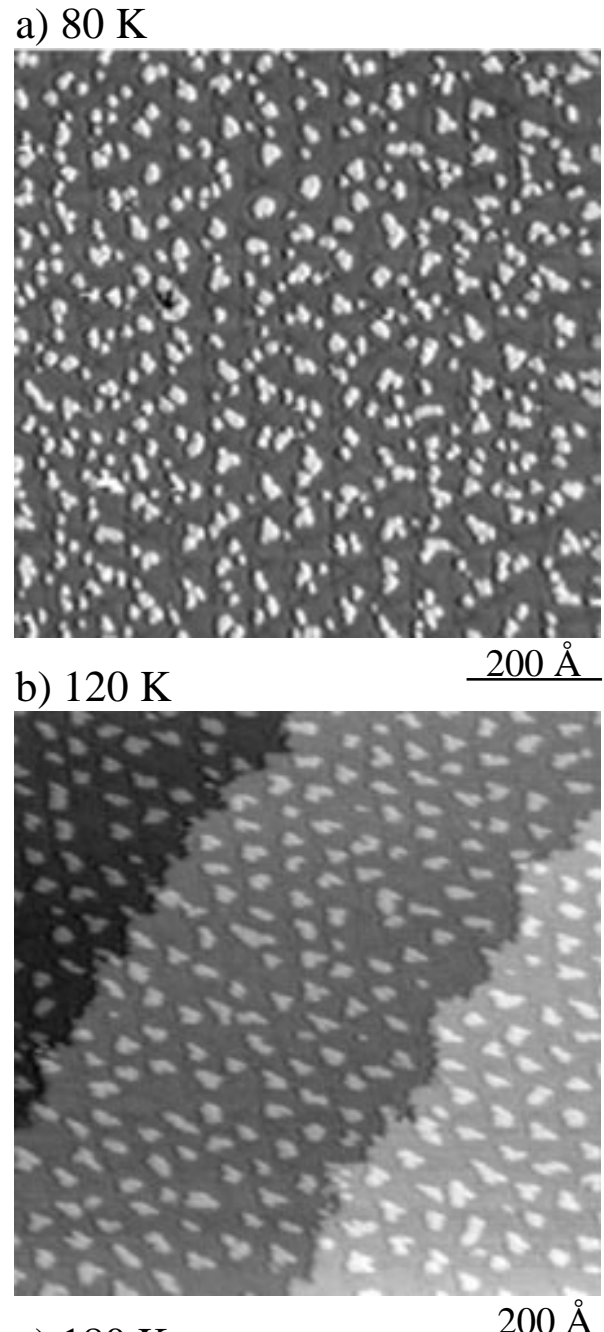

c) $180 \mathrm{~K}$

$200 \AA$

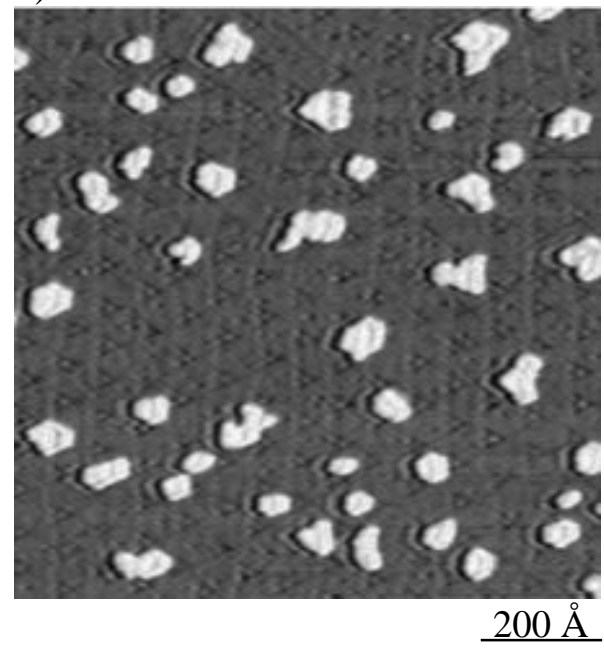

Fig. 2. Temperature series of Ag nucleation on the dislocation network of Fig. 1a. (a) At temperatures below $100 \mathrm{~K}$ more than one island nucleates within each unit cell. (b) At temperatures between $100 \mathrm{~K}$ and $130 \mathrm{~K}$, ordered nucleation and formation of a superlattice is found. (c) At temperatures above $130 \mathrm{~K}$ diffusing adatoms can overcome the repulsive dislocations and the island density drops below one island per unit cell. Ag coverage $\theta=0.1 \mathrm{ML}$. 


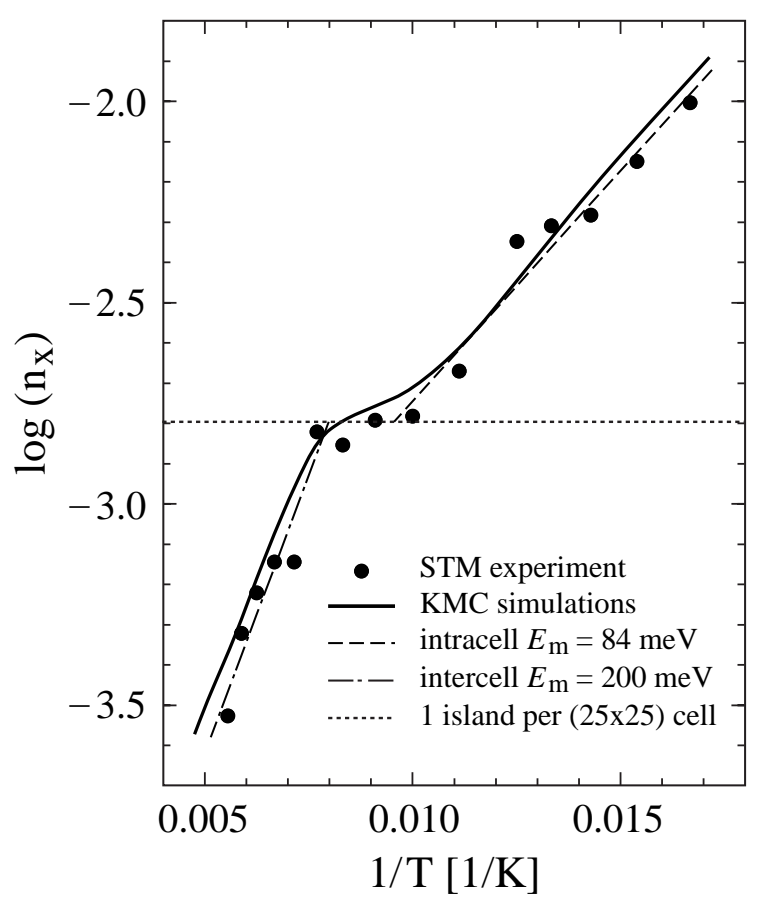

Fig. 3. Arrhenius plot of measured and simulated island densities for nucleation of $0.1 \mathrm{ML} \mathrm{Ag}$ on the dislocation network shown in Fig. 1a. The island densities clearly show two slopes separated by a plateau located at a density of one island per superstructure unit cell. At temperatures below this plateau, the mean free path of $\mathrm{Ag}$ adatoms is smaller than the $(25 \times 25)$ unit cell leading to nucleation of several islands per unit cell. At the plateau, the diffusivity becomes sufficient for the adatoms to explore the entire unit cell on the time scale of deposition, but their thermal energy is not yet sufficient to surmount the barrier represented by the dislocations. Therefore the adatoms are confined to the unit cell into which they were deposited and nucleate one island there. Eventually, the barrier at dislocations can be overcome and the island density further decreases. The full line shows results from the kinetic Monte-Carlo simulations.

fcc-areas, in agreement to theory [13]. Hence, atoms landing in one of the two hcp triangles can diffuse into the fcc hexagons but not vice versa. Due to the repulsive nature of the dislocations and the attraction towards specific sites within the unit cell, the periodicity of the dislocation network is congruently transferred to an island superlattice. For growth temperatures of $140 \mathrm{~K}$ or above, the island density decreases again. Here, the adatom's thermal energy is sufficient to overcome the repulsive barriers and the $\mathrm{Ag}$ atoms diffuse over several superstructure cells before nucleating (Fig. 2c).

In an Arrhenius representation of the island densities both the low temperature and the high temperature region are characterized by straight lines (Fig. 3). The rate-limiting processes of island nucleation that define the slopes of these lines are the intracell diffusion of adatoms within the supercells of the dislocation network at low temperature, and the intercell diffusion over the dislocations at high temperature. To obtain a quantitative understanding of the activation energies for these diffusion processes, we a)

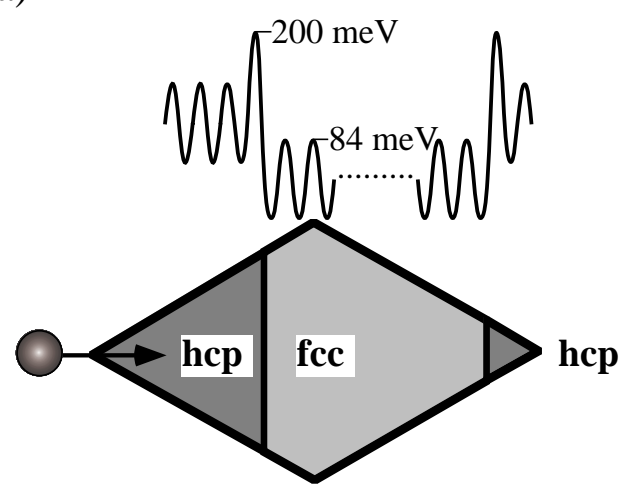

b)

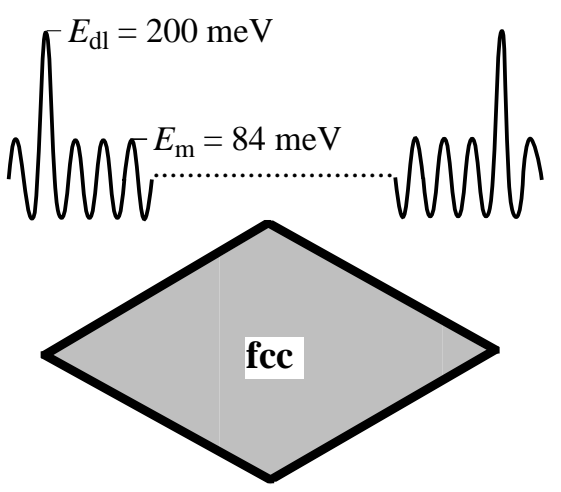

Fig. 4. Model of the kinetic Monte-Carlo simulations. The complicated $(25 \times 25)$ unit cell of the superstructure with one fcc and two different hcp domains, the latter with a higher energy for diffusing adatoms (a), was modeled as one uniform domain of constant activation energy $E_{\mathrm{m}}=84 \pm 8 \mathrm{meV}$, separated from the adjacent unit cells by a diffusion barrier of $E_{\mathrm{dl}}=200 \pm 30 \mathrm{meV}$.

performed kinetic Monte-Carlo simulations on a hexagonal lattice with a $(25 \times 25)$ superstructure. For simplicity we did not distinguish between fcc and hcp sites, hence the unit cell was a simple rhombus, surrounded by the dislocations acting as repulsive line defects. The free parameters are the energy barriers for diffusion within $\left(E_{\mathrm{m}}\right)$ and across $\left(E_{\mathrm{dl}}\right)$ the unit cells (see Fig. 4b). The simulation clearly shows the transition between two slopes. It yields $E_{\mathrm{m}}=$ $84 \pm 8 \mathrm{meV}$ for intracell diffusion and $E_{\mathrm{dl}}=200 \pm 30 \mathrm{meV}$ for diffusion across dislocations. The strong repulsion at dislocations explains the extended temperature regime of ordered nucleation. The barrier of intracell diffusion lies between those measured for $\mathrm{Ag}(111)$ self-diffusion and $\mathrm{Ag}$ diffusion on the coherently strained, pseudomorphic first Ag layer on $\mathrm{Pt}(111), E_{\mathrm{m}}=100 \mathrm{meV}$ and $E_{\mathrm{m}}=60 \mathrm{meV}$, respectively [14]. This result could be expected, because the diffusion energy generally decreases with increasing strain in the system [14]. In the fcc and hcp patches of the second layer the compressive strain is less than in the first layer, but it is not yet entirely relieved.

The region that is of most interest for the self-organized growth of ordered nanostructure arrays is the plateau where the substrate superstructure is congruently trans- 
ferred to a cluster array. This plateau is less pronounced in our Monte-Carlo simulations than in the experiment. In reality, dislocations have a long range repulsion towards adatoms, funneling them towards the center of the unit cell where island nucleation takes place. We did not attempt to simulate the complicated adsorption energy surface required to account for these findings, and consequently the islands in the simulation are randomly placed within the unit cells which smears out the plateau. However, we find from a series of simulations that the width of the plateau is exclusively determined by the ratio of the two barriers, $E_{\mathrm{m}}$ and $E_{\mathrm{dl}}$. Systems revealing a stronger repulsion at dislocations have an extended temperature regime of highly ordered nucleation.

An interesting side-effect of the ordered nucleation in the intermediate temperature range is an enhanced size uniformity. Atoms deposited into a network unit cell stay confined to this cell by the surrounding dislocations. In the limit of completely repulsive edges of the supercells, sufficiently high mobility of the atoms to ensure nucleation of only one island, and not too high coverages which guaranties that the deposition processes into different supercells are independent events, the size distribution of the islands (number of atoms in the islands) is binomial, $P_{k}=\left(\begin{array}{l}n \\ k\end{array}\right) p^{k} q^{n-k}$ (with $p=\theta, q=1-\theta, k$ being the island size, $n$ the size of the unit cell, and $\theta$ the coverage). For our case of $p=0.1$ and $n=625$ the binomial distribution has a relative standard deviation (normalized to the average island size) of $\sigma=(q / n p)^{1 / 2}=0.12$. Due to the convolution of tip and island shape there is a residual width, which explains our larger experimental value of $\sigma=0.20$ [10]. This value represents an upper bound to the real size distribution. The formula of the relative standard deviation evinces the potential of the method: In a system with a superstructure unit cell as large as in the present case, and a coverage of $\theta=0.5 \mathrm{ML}$, one expects a relative width of the size distribution of only $4 \%$ in cluster area.

Our experiments describe a new method to create almost monodispersed, equally spaced nanostructures through self-organization on a template with a periodic network of dislocations. The inhomogeneous potential en- ergy surface experienced by diffusing adatoms gives rise to heterogeneous nucleation at well defined sites within the superstructure and therefore to well ordered cluster arrays. We showed how to map out this energy surface for our particular example. Similar effects of self-organization can be expected for a wide range of epitaxial systems. This approach thus provides a method of general potential for the fabrication of quantum arrays, i.e., superlattices of nanostructures with size and period smaller than the Fermi wave-length.

\section{References}

1. D.M. Eigler, E.K. Schweizer: Nature 344, 524 (1990)

2. H. Röder, E. Hahn, H. Brune, J.P. Bucher, K. Kern: Nature 366, 141 (1993)

3. D. Leonard, M. Krishnamurthy, C.M. Reaves, S.P. Denbaars, P.M. Petroff: Appl. Phys. Lett. 63, 3203 (1993)

4. R. Nötzel, J. Tommyo, T. Tamamura: Nature 369, 131 (1994)

5. J.M. Moison, F. Houzay, F. Barthe, L. Leprince, E. André, O. Vatel: Appl. Phys. Lett. 64, 196 (1994)

6. K. Bromann, C. Félix, H. Brune, W. Harbich, R. Monot, J. Buttet, K. Kern: Science 274, 956 (1996)

7. J. Tersoff, C. Teichert, M.G. Lagally: Phys. Rev. Lett 76, 1675 (1996)

8. D.D. Chambliss, R.J. Wilson, S. Chiang: Phys. Rev. Lett. 66, 1721 (1991)

9. J.A. Meyer, J.D. Baikie, E. Kopatzki, R.J. Behm: Surf. Sci. 365, L647 (1996)

10. H. Brune, M. Giovannini, K. Bromann, K. Kern: Nature 394, 451 (1998)

11. H. Brune, H. Röder, K. Bromann, K. Kern: Thin Solid Films 264, 230 (1995)

12. H. Brune, H. Röder, C. Boragno, K. Kern: Phys. Rev. B 49, 2997 (1994)

13. C. Ratsch, A.P. Seitsonen, M. Scheffler: Phys. Rev. B 55, 6750 (1997)

14. H. Brune, K. Bromann, H. Röder, K. Kern, J. Jacobsen, P. Stolze, K. Jacobsen, J. Nørskov: Phys. Rev. B 52, R14380 (1995) 